\title{
PERIODIC BURSTS OF COHERENT RADIO EMISSION FROM AN ULTRACOOL DWARF
}

\author{
G. Hallinan, ${ }^{1}$ S. Bourke, ${ }^{1}$ C. Lane, ${ }^{1}$ A. Antonova, ${ }^{2}$ R. T. Zavala, ${ }^{3}$ W. F. Brisken, ${ }^{4}$ R. P. Boyle, ${ }^{5}$ \\ F. J. VRba, ${ }^{3}$ J. G. Doyle, ${ }^{2}$ AND A. Golden ${ }^{1}$ \\ Received 2007 February 20; accepted 2007 May 14; published 2007 June 15
}

\begin{abstract}
We report the detection of periodic $(p=1.96 \mathrm{hr})$ bursts of extremely bright, $100 \%$ circularly polarized, coherent radio emission from the M9 dwarf TVLM 513-46546. Simultaneous photometric monitoring observations have established this periodicity to be the rotation period of the dwarf. These bursts, which were not present in previous observations of this target, confirm that ultracool dwarfs can generate persistent levels of broadband, coherent radio emission, associated with the presence of $\mathrm{kG}$ magnetic fields in a large-scale, stable configuration. Compact sources located at the magnetic polar regions produce highly beamed emission generated by the electron cyclotron maser instability, the same mechanism known to generate planetary coherent radio emission in our solar system. The narrow beams of radiation pass our line of sight as the dwarf rotates, producing the associated periodic bursts. The resulting radio light curves are analogous to the periodic light curves associated with pulsar radio emission highlighting TVLM 513-46546 as the prototype of a new class of transient radio source.
\end{abstract}

Subject headings: pulsars: general — radio continuum: stars — radiation mechanisms: nonthermal — stars: activity — stars: low-mass, brown dwarfs — stars: magnetic fields

\section{INTRODUCTION}

Ultracool dwarfs are generally defined as those dwarfs with spectral type $\geq M 7$ (Kirkpatrick et al. 1997), encompassing very low mass stars just above the stellar/substellar boundary and all brown dwarfs below it. Surprisingly, despite their low bolometric luminosity, a number of ultracool dwarfs have been detected as relatively bright sources at radio frequencies (Berger et al. 2001; Berger 2002, 2006; Burgasser \& Putman 2005; Phan-Bao et al. 2007). Initially, the detection of variable, broadband radio emission with low net circular polarization suggested incoherent gyrosynchrotron radiation due to a population of electrons spiraling in the magnetic field of the ultracool dwarf (Berger et al. 2005; Osten et al. 2006). This is the mechanism by which higher mass dwarf stars are thought to generate persistent levels of radio emission. However, such stars are surrounded by high-temperature chromospheres and coronae, and the levels of radio emission they produce have been found to be tightly correlated to their coronal X-ray luminosities (Güdel \& Benz 1993; Benz \& Güdel 1994). Ultracool dwarfs, on the other hand, are thought to possess cooler, more neutral atmospheres, as evidenced by their low $\mathrm{H} \alpha$ and $\mathrm{X}$-ray luminosities (Mohanty \& Basri 2003; Stelzer et al. 2006) and yet can produce high levels of radio emission, many orders of magnitude brighter than those predicted by this relationship.

Berger et al. (2005) reported a periodicity of $\sim 3 \mathrm{hr}$ in the radio emission detected from one such ultracool dwarf 2MASS J00361617+1821104, citing orbital motion of a companion, rotation of the dwarf, or periodic magnetic reconnection as possible causes of the observed behavior. Hallinan et al. (2006) detected

\footnotetext{
${ }^{1}$ Computational Astrophysics Laboratory, National University of Ireland, Galway, Ireland; gregg@it.nuigalway.ie, stephen@it.nuigalway.ie, c.lane2@ nuigalway .ie, agolden@it.nuigalway.ie.

${ }^{2}$ Armagh Observatory, Armagh BT61 9DG, North Ireland; tan@arm.ac.uk, jgd@arm.ac.uk.

${ }^{3}$ US Naval Observatory, Flagstaff Station, Flagstaff, AZ 86001; bzavala@nofs.navy.mil, fjv@nofs.navy.mil.

${ }^{4}$ National Radio Astronomy Observatory, Socorro, NM 87801; wbrisken@aoc .nrao.edu.

${ }^{5}$ Vatican Observatory Research Group, Steward Observatory, University of Arizona, Tucson, AZ 85721; boyle@ ricci.as.arizona.edu.
}

a periodicity in the radio emission from a second ultracool dwarf, TVLM 513-46546 (hereafter TVLM 513), consistent with the putative rotation period of the dwarf derived from $v \sin i$ data, thereby favoring rotation as the cause of the observed periodicities for both dwarfs. On the basis of this assumption, the periodic variability can be explained by either (1) an anisotropically beamed radio emission mechanism or (2) the occultation of very high brightness temperature compact regions in the magnetosphere of the dwarf. Both scenarios were deemed inconsistent with isotropic gyrosynchrotron emission from a large extended corona. It was postulated that the radio emission from ultracool dwarfs may instead be due to coherent electron cyclotron maser emission generated in the low-density regions above the poles of a large-scale magnetic field (Hallinan et al. 2006), the same mechanism producing the coherent radiation detected from the magnetized planets in our solar system (Zarka 1998; Ergun et al. 2000). However, studies of the electron cyclotron maser instability operating in planetary magnetospheres have reported intrinsically narrow banded, $100 \%$ polarized emission. The radio emission from TVLM 513, on the other hand, was found to be broadband with net circular polarization $\leqslant 13 \%$ reaching a periodic maximum of $\sim 40 \%$, properties more consistent with the incoherent gyrosynchrotron process. We have conducted further deep pointings of TVLM 513 in an effort to unambiguously distinguish between incoherent gyrosynchrotron emission and coherent electron cyclotron maser emission. The periodicity reported by Hallinan et al. (2006) was once again present, but the dwarf was found to be in a much more active state than in previous epochs, producing periodic bursts of extremely bright $100 \%$ circularly polarized emission. These observations provide direct confirmation that ultracool dwarfs can produce persistent levels of broadband, $100 \%$ polarized, coherent electron cyclotron maser emission.

\section{OBSERVATIONS}

We used the Very Large Array (VLA) ${ }^{6}$ to observe the M9 dwarf TVLM 513-46546 for $\sim 10 \mathrm{hr}$ at a frequency of $8.44 \mathrm{GHz}$ on

\footnotetext{
${ }^{6}$ The VLA is operated by the National Radio Astronomy Observatory, a facility of the National Science Foundation operated under cooperative agreement by Associated Universities, Inc.
} 

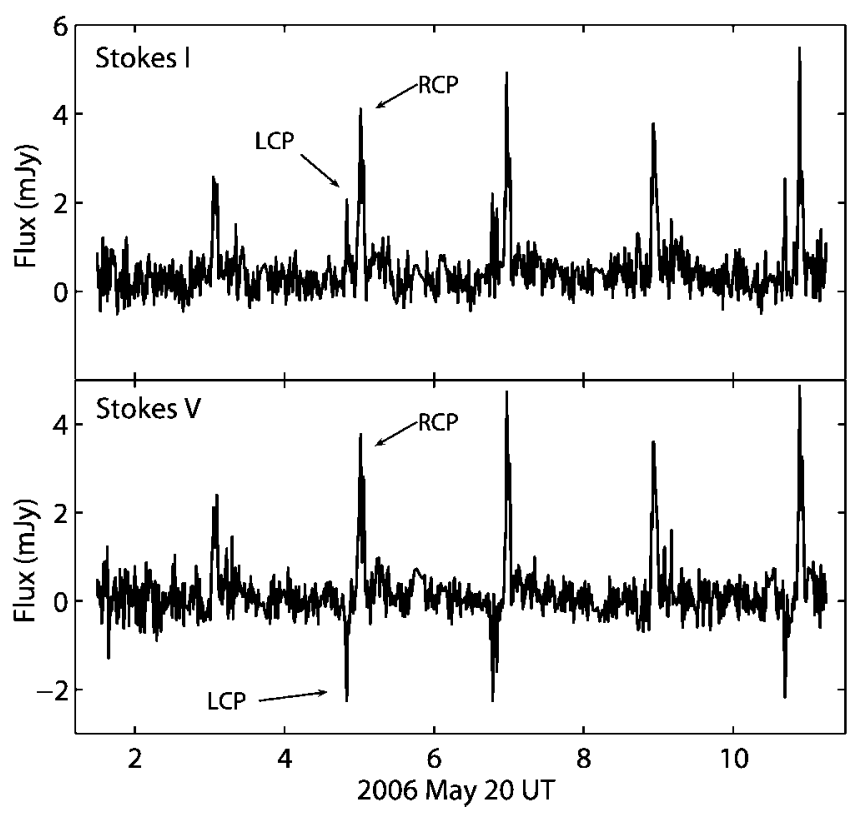

FIG. 1.-Light curves of the total intensity (Stokes $I$ ) and the circularly polarized (Stokes $V$ ) radio emission detected at $8.44 \mathrm{GHz}$ from TVLM 513. Right circular polarization is represented by positive values, and left circular polarization is represented by negative values in the Stokes $V$ light curve. Bursts of both $100 \%$ right circularly polarized emission (an example is highlighted as RCP) and 100\% left circularly polarized emission (an example is highlighted as LCP) are detected with a periodicity of $1.96 \mathrm{hr}$. The absence of a left circularly polarized burst at $\sim 8.7$ UT is due to the VLA being pointed at a phase calibration source.

2006 May 20 and for $\sim 10 \mathrm{hr}$ at a frequency of $4.88 \mathrm{GHz}$ on 2006 May 21. Data reduction was carried out with the Astronomical Image Processing System (AIPS) software package. The light curve of the $8.44 \mathrm{GHz}$ radio emission is characterized by periodic bursts of both left and right $100 \%$ circularly polarized emission, the brightest periodically reaching flux values of $\sim 5 \mathrm{mJy}$, which are stable in phase for the duration of the observation (Fig. 1). Similar, albeit less bright (up to $\sim 1 \mathrm{mJy}$ ), periodic bursts are also present in the $4.88 \mathrm{GHz}$ light curve. The periodicity of $1.96 \mathrm{hr}$ was also retrieved in I-band photometric monitoring observations of TVLM 513 using telescopes at the US Naval Observatory (USNO), Flagstaff Station, and the Vatican Observatory (VO), Arizona confirming that the periodicity present in the radio emission from TVLM 513 is due to the rotation of the dwarf (Fig. 2). Therefore, although the bright, polarized bursts present in the radio data appear to be transient flares, they are produced by the strong beaming of persistent radio emission together with the rapid rotation of the dwarf. For the limiting case of perfectly beamed emission and assuming a source $<0.5 R$ from the surface, where $\mathrm{R}$ is the radius of the dwarf, we can use the duration of the bursts to geometrically constrain the size of the emitting source region to be $<0.22 R$. Using this value, we can establish the brightness temperature of the radio emission to be $>2.4 \times 10^{11} \mathrm{~K}$, which, together with the $100 \%$ circular polarization and extremely narrow beaming, provides conclusive confirmation of its coherent nature.

The phase-folded light curves of the radio emission detected from TVLM 513 at 8.44 and $4.88 \mathrm{GHz}$ are shown in Figure 3. The flux detected over the duration of the observation was $0.464 \pm 0.009$ and $0.368 \pm 0.011 \mathrm{mJy}$ at 8.44 and $4.88 \mathrm{GHz}$, respectively. These values are similar to those reported previously for this dwarf by Hallinan et al. (2006) of $0.405 \pm$ 0.018 and $0.396 \pm 0.016 \mathrm{mJy}$ at 8.44 and $4.88 \mathrm{GHz}$, respectively. However, the periodic bursts, particularly those detected

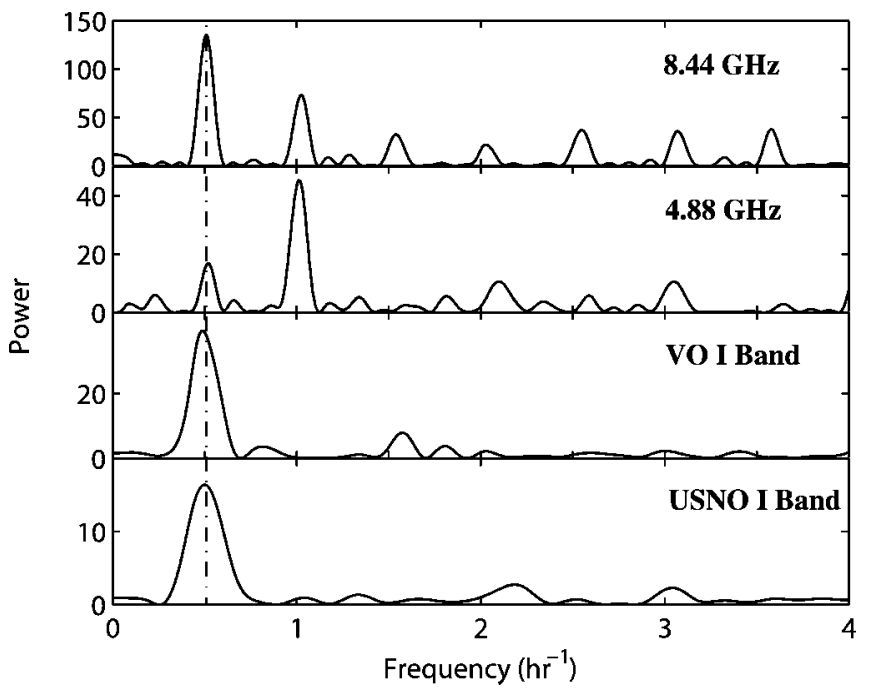

FIG. 2.-Lomb-Scargle periodograms of (a) the $8.44 \mathrm{GHz}$ radio data obtained on 2006 May 20, (b) the $4.88 \mathrm{GHz}$ radio data obtained on 2006 May $21,(c)$ the $I$-band photometric monitoring data obtained on 2006 May 21 with the VO $1.8 \mathrm{~m}$ telescope, and $(d)$ the $I$-band photometric monitoring data obtained on 2006 May 18 with the USNO Flagstaff Station $1 \mathrm{~m}$ telescope. The dashed line corresponds to the putative rotational period of $1.96 \mathrm{hr}$ for TVLM 513. The periodicity present in the radio data is also present in the optical data confirming rotation of the dwarf as the source of this periodicity.

at $8.44 \mathrm{GHz}$, which are an order of magnitude brighter than the mean flux values, were not detected in the observations of Hallinan et al. (2006), indicating that activity levels in the compact regions producing the bursts have increased greatly in the intervening $\sim 16$ months between the two epochs. Although the
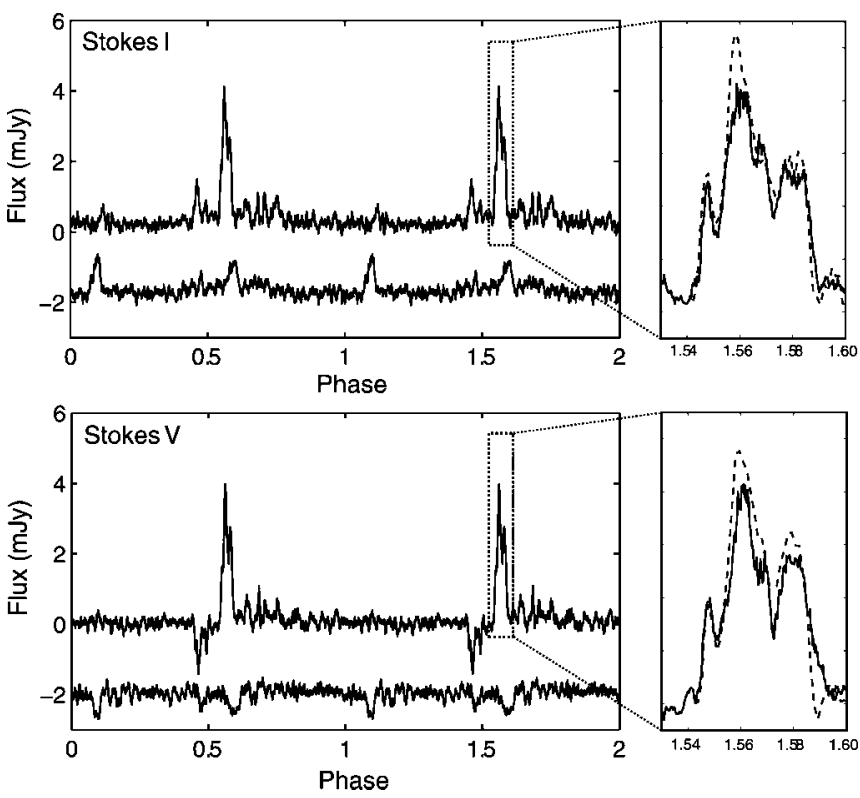

FIG. 3.- Light curves of the total intensity (Stokes $I$ ) and circularly polarized (Stokes $V$ ) radio emission detected from TVLM 513 at 8.44 and $4.88 \mathrm{GHz}$ ( $4.88 \mathrm{GHz}$ shifted by $-2 \mathrm{mJy}$ ) phase-folded to a period of $1.958 \mathrm{hr}$. For the circularly polarized (Stokes $V$ ) light curves, right circular polarization is represented by positive values, and left circular polarization is represented by negative values. Two periods of each phase-folded light curve are shown for clarity. Inset: Closer examination of the brightest burst detected at $8.44 \mathrm{GHz}$ reveals further structure, possibly due to the anisotropic morphology of the source region of this burst. The dashed line corresponds to an unfolded light curve of one of the bursts correlated with the phase-folded light curve to confirm that this further structure is not a product of the phase folding of the data. 
periodic bursts are $100 \%$ circularly polarized, the interpulse emission at both frequencies is largely unpolarized. This unpolarized component does not display the high degree of periodic variation observed for the $100 \%$ circularly component of the emission, indicating much lower intrinsic beaming of the emission.

Although periodic bursts are detected at 4.88 and $8.44 \mathrm{GHz}$, the phase-folded light curves suggest narrowband structure across this frequency range, for example, the brightest burst in the 8.44 GHz phase-folded light curve does not appear to have a counterpart in the $4.88 \mathrm{GHz}$ light curve. Although there is a burst at a similar phase in the $4.88 \mathrm{GHz}$ light curve, it is left circularly polarized and thus probably originates in a region of opposite magnetic polarity to the right circularly polarized $8.44 \mathrm{GHz}$ burst. It should be noted that direct correlation of the radio emission at both frequencies is subject to the assumption that the periodic bursts were stable in phase and amplitude over the duration of the two night monitoring campaign, as data were not obtained at both frequencies simultaneously. It is notable that the phase-folded light curve of the $4.88 \mathrm{GHz}$ emission is characterized by two highly polarized bursts per period of rotation of the dwarf, separated by $\sim 0.5$ phase. This is consistent with emission from the same source region emitted perpendicular to the local magnetic field and hence detected twice per period of rotation. In contrast, the bursts observed at $8.44 \mathrm{GHz}$ are only detected once per rotation and hence may be obscured on one side by optically thick plasma or indeed the stellar surface.

\section{THE ELECTRON CYCLOTRON MASER}

The characteristics of the periodic bursts, which include $100 \%$ circular polarization, high brightness temperature, and very narrow beaming, are all consistent with coherent emission generated via the electron cyclotron maser instability (Treumann 2006). As discussed by Hallinan et al. (2006), the unpolarized component of the radio emission detected from TVLM 513 may also be produced by the coherent electron cyclotron maser mechanism, but incoherent gyrosynchrotron or synchrotron emission cannot be ruled out without further multifrequency observations to separately characterize the $100 \%$ circularly polarized bursts and the unpolarized component of the emission.

The electron cyclotron maser instability is the mechanism deemed responsible for the coherent radio emission detected from the magnetized planets in our solar system (Zarka 1998; Ergun et al. 2000) and has also been proposed as a source of certain classes of solar and stellar bursts. The bulk of planetary electron cyclotron maser emission is confined to high magnetic latitudes, and in situ measurements of Earth's auroral kilometric radiation (AKR) have provided the deepest insights in to the nature of the radiation mechanism. AKR is associated with the presence of magnetic field-aligned electric fields at high magnetic latitudes that (1) evacuate thermal plasma along open field lines, enabling the formation of density cavities where the electron cyclotron frequency can greatly exceed the plasma frequency, $\nu_{c} \gg \nu_{p}$, a prerequisite for the generation of electron cyclotron maser emission and (2) accelerate electrons into these density cavities that adiabatically evolve along field lines of increasing strength to form an unstable "horseshoe" or "shell" distribution (Pritchett 1984; Pritchett \& Strangeway 1985; Winglee \& Pritchett 1986), providing the free energy to power the maser emission. Emission in the R-X mode perpendicular to the magnetic field in the source region is favored, resulting in emission of opposite polarity from magnetic polar regions of opposite polarity. A similar model was proposed by Hallinan et al. (2006) to account for the radio emission detected from ultracool dwarfs, now confirmed to be the case with the detection of periodic $100 \%$ circularly polarized bursts from TVLM 513.

The means by which the stable magnetic field aligned electric fields are generated and maintained in the magnetosphere of TVLM 513 remains unclear. We rule out the interaction of the magnetosphere with a stellar wind from a coronally active star, the means by which a large fraction of planetary maser emission is powered, as high-resolution adaptive optics observations of TVLM 513 have found no evidence of a physical companion (Close et al. 2003). It is also unlikely that a close-in orbital companion is responsible, as is the case for the component of Jupiter's maser emission associated with the Jupiter-Io electrodynamic circuit. The narrow duty cycle bursts detected from TVLM 513 are tightly locked in phase with rotation of the dwarf and hence are not in corotation with an orbital companion. We can assert therefore that the stable electric fields are somehow generated and sustained within the magnetosphere of the ultracool dwarf. It is worth noting that the rapid rotation of Jupiter is also thought to be an efficient source of electron acceleration for a component of the non-Io related maser emission and that a similar scenario may be applicable to TVLM 513.

\section{MAGNETIC FIELD DIAGNOSTICS}

Electron cyclotron maser radiation is emitted at the electron cyclotron frequency denoted by $\nu_{c} \approx 2.8 \times 10^{6} B \mathrm{~Hz}$, where $B$ is the magnetic field strength in the radio emission source region. It has been proposed that emission at the secondary harmonic may dominate where radiation generated at the fundamental electron cyclotron frequency cannot escape due to gyroharmonic absorption by thermal plasma, for example, in the case of maser emission generated at the base of magnetic flux tubes in the solar corona (Melrose \& Dulk 1982). However, thermal plasma density is expected to be much lower in the density cavities located at the magnetic polar regions of TVLM 513 than at the base of the solar corona, and therefore optical depth at the secondary harmonic layer should also be much smaller than that derived for the solar case. Melrose \& Dulk (1982) also note that growth at the fundamental is extremely efficient, limiting the energy available for growth at higher harmonics. Importantly, measurements by the FAST satellite at Earth and the Ulysses probe at Jupiter have directly confirmed that planetary electron cyclotron maser emission is almost entirely generated at the fundamental frequency. Therefore, emission at the fundamental frequency is strongly favored for the bursts of electron cyclotron maser emission detected from TVLM 513. We can thus derive the field strength at the source of the $8.44 \mathrm{GHz}$ bursts detected from TVLM 513 to be $\sim 3 \mathrm{kG}$, confirming the estimate of Hallinan et al. (2006). These high magnetic field strengths, which can also be attributed to other ultracool dwarfs previously detected at radio frequencies including a number of bona fide brown dwarfs, are of the same order as those confirmed for the most magnetically active dMe flare stars (Johns-Krull \& Valenti 1996). Reiners \& Basri (2007) have recently directly measured the magnetic field strengths of a number of ultracool dwarfs, confirming that magnetic flux is indeed maintained at the lower end of the main sequence.

The extremely high stability in phase of the narrow duty cycle, $100 \%$ circularly polarized bursts detected from TVLM 513, coupled with the detection of bursts at both 4.88 and $8.44 \mathrm{GHz}$, confirm that these high-strength magnetic fields are in a large- 
scale, stable configuration. This is further supported by the periodic signal detected in the $I$-band photometric data (Fig. 2), which is stable in phase and amplitude for the duration of the four night monitoring campaign, consistent with the presence of high-strength, fixed magnetic field regions at the stellar photosphere (C. Lane et al. 2007, in preparation). Multiple bursts of both left and right $100 \%$ circularly polarized emission, which originate in regions of opposite magnetic polarity, are confined to the same range of phase of rotation of the dwarf $(\sim 0.45-0.78$; Fig. 3), indicating the probable presence of a dipolar component to this large-scale magnetic field. Periodic bursts are detected when the axis of this dipolar field lies in the plane of the sky, which occurs twice per period of rotation of the dwarf. This model requires the dipolar field to be tilted relative to the rotation axis of the dwarf.

\section{DISCUSSION AND CONCLUSIONS}

Electron cyclotron maser emission generated at the poles of a large-scale magnetic field may be a ubiquitous source of radio emission from planets, brown dwarfs and very low mass stars. Kellett et al. (2002) and Bingham et al. (2001) have also proposed that electron cyclotron maser emission originating in the polar regions of a large-scale magnetic field may be a source of radio emission from coronally active stars, particularly dMe flare stars. The recent magnetic mapping of a rapidly rotating M4 dwarf has revealed the presence of a dipolar component, confirming that such stars can indeed possess large-scale, stable magnetic fields (Donati et al. 2006). In light of the observed behavior of TVLM 513 we note that many of the coherent bursts detected from stellar sources, particularly dMe flare stars, might not be transient flare events but rather may be due to the beaming of persistent electron cyclotron maser emission coupled with rotation of the star. Periodicity may have gone undetected for higher mass stars as they have much longer periods of rotation than ultracool dwarfs (Zapatero Osorio et al. 2006), making it difficult to directly associate coherent flares with a particular phase of rotation.

In light of the sharp drop observed in the levels of $\mathrm{H} \alpha$ and
X-ray emission for late M, L, and T dwarfs, the electron cyclotron maser may prove a vital diagnostic tool for remote sensing of plasma conditions and magnetic field strengths and topologies in the mass gap between planets and stars. Previous radio surveys have been undertaken of a volume-limited sample of ultracool dwarfs in the solar neighborhood, resulting in an overall detection rate of $\sim 10 \%$ (Berger 2006). However, these surveys were conducted solely at a frequency of $8.44 \mathrm{GHz}$, important when considering that electron cyclotron maser emission is characterized by an upper cutoff frequency associated with the maximum magnetic field strength in the magnetosphere of the dwarf. A detection at $8.44 \mathrm{GHz}$ requires magnetic field strengths of at least $3 \mathrm{kG}$. Therefore, a much larger fraction of these objects with maximum field strengths below this value, particularly lower mass $\mathrm{L}$ and $\mathrm{T}$ dwarfs, may be detectable sources at lower frequencies. We highlight the recent observation of periodic bursts of coherent emission from an unknown source toward the Galactic center, GCRT J1745-3009 (Hyman et al. 2005), at a frequency of $330 \mathrm{MHz}$, as the possible detection of an as yet unidentified cool, dim brown dwarf or extrasolar planet in the solar neighborhood. In particular, the period of $1.28 \mathrm{hr}$ observed for the bursts from GCRT J1745-3009 compares favorably with the period of $0.98 \mathrm{hr}$ observed for the bursts detected at $4.88 \mathrm{GHz}$ from TVLM 513, and the bursts have only been detected in $\sim 7 \%$ of the time spent on source thus far (Hyman et al. 2007), indicating longterm activity variation similar to that observed for TVLM 513.

We gratefully acknowledge the support of the HEA funded Cosmogrid project, the Irish Research Council for Science, Engineering, and Technology (IRCSET), and Enterprise Ireland. A. G. is supported by Science Foundation Ireland (grant 07/RFP/PHYF553). Armagh Observatory is grant-aided by the N. Ireland Department of Culture, Arts, and Leisure. We would like to thank Ray Butler, James Mc Donald, and Evan Keane for assistance with certain aspects of this manuscript and the referee Manuel Güdel, whose extensive comments significantly improved this manuscript.

\section{REFERENCES}

Benz, A. O., \& Güdel, M. 1994, A\&A, 285, 621

Berger, E. 2002, ApJ, 572, 503

$$
\text { 2006, ApJ, 648, } 629
$$

Berger, E., et al. 2001, Nature, 410, 338 2005, ApJ, 627, 960

Bingham, R., Cairns, R. A., \& Kellett, B. J. 2001, A\&A, 370, 1000

Burgasser, A. J., \& Putman, M. E. 2005, ApJ, 626, 486

Close, L. M., Siegler, N., Freed, M., \& Biller, B. 2003, ApJ, 587, 407

Donati, J.-F., Forveille, T., Cameron, A. C., Barnes, J. R., Delfosse, X., Jardine, M. M., \& Valenti, J. A. 2006, Science, 311, 633

Ergun, R. E., Carlson, C. W., McFadden, J. P., Delory, G. T., Strangeway, R. J., \& Pritchett, P. L. 2000, ApJ, 538, 456

Güdel, M., \& Benz, A. O. 1993, ApJ, 405, L63

Hallinan, G., Antonova, A., Doyle, J. G., Bourke, S., Brisken, W. F., \& Golden, A. 2006, ApJ, 653, 690

Hyman, S. D., Lazio, T. J. W., Kassim, N. E., Ray, P. S., Markwardt, C. B., \& Yusef-Zadeh, F. 2005, Nature, 434, 50

Hyman, S. D., Subhashis, R., Sabyasachi, P., Lazio, T. J. W., Ray, P. S., Kassim, N. E., \& Bhatnagar, S. 2007, ApJ, submitted (astro-ph/0701098)
Johns-Krull, C. M., \& Valenti, J. A. 1996, ApJ, 459, L95

Kellett, B. J., Bingham, R., Cairns, R. A., \& Tsidouki, V. 2002, MNRAS, 329, 102

Kirkpatrick, J. D., Henry, T. J., \& Irwin, M. J. 1997, AJ, 113, 1421

Melrose, D. B., \& Dulk, G. A. 1982, ApJ, 259, 844

Mohanty, S., \& Basri, G. 2003, ApJ, 583, 451

Osten, R. A., Hawley, S. L., Bastian, T. S., \& Reid, I. N. 2006, ApJ, 637, 518

Phan-Bao, N., Osten, R. A., Lim, J., Martín, E. L., \& Ho, P. T. P. 2007, ApJ, 658,553

Pritchett, P. L. 1984, J. Geophys. Res., 89, 8957

Pritchett, P. L., \& Strangeway, R. J. 1985, J. Geophys. Res., 90, 9650

Reiners, A., \& Basri, G. 2007, ApJ, 656, 1121

Stelzer, B., Micela, G., Flaccomio, E., Neuhäuser, R., \& Jayawardhana, R. 2006, A\&A, 448, 293

Treumann, R. A. 2006, A\&A Rev., 13, 229

Winglee, R. W., \& Pritchett, P. L. 1986, J. Geophys. Res., 91, 13531

Zapatero Osorio, M. R., Martín, E. L., Bouy, H., Tata, R., Deshpande, R., \& Wainscoat, R. 2006, ApJ, 647, 1405

Zarka, P. 1998, J. Geophys. Res., 103, 20159 